NIST Technical Note 2144

\title{
Evaluation of Timers Related to ProSe-based UE-to-Network Relays
}

Aziza Ben Mosbah

Samantha Gamboa

Richard Rouil

This publication is available free of charge from:

https://doi.org/10.6028/NIST.TN.2144

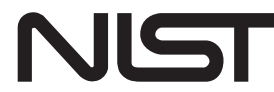

National Institute of Standards and Technology

U.S. Department of Commerce 


\title{
NIST Technical Note 2144
}

\section{Evaluation of Timers Related to ProSe-based UE-to-Network Relays}

\author{
Aziza Ben Mosbah \\ Samantha Gamboa \\ Associate, Wireless Networks Division \\ Communications Technology Laboratory \\ Prometheus Computing LLC \\ Sylva, North Carolina \\ Richard Rouil \\ Wireless Networks Division \\ Communications Technology Laboratory
}

This publication is available free of charge from:

https://doi.org/10.6028/NIST.TN.2144

March 2021

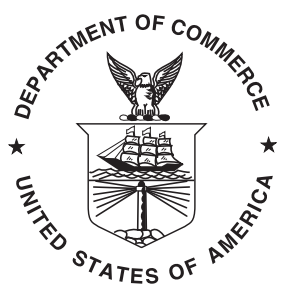

U.S. Department of Commerce Gina M. Raimondo, Secretary

National Institute of Standards and Technology James K. Olthoff, Performing the Non-Exclusive Functions and Duties of the Under Secretary of Commerce for Standards and Technology \& Director, National Institute of Standards and Technology 
Certain commercial entities, equipment, or materials may be identified in this document in order to describe an experimental procedure or concept adequately. Such identification is not intended to imply recommendation or endorsement by the National Institute of Standards and Technology, nor is it intended to imply that the entities, materials, or equipment are necessarily the best available for the purpose.

National Institute of Standards and Technology Technical Note 2144

Natl. Inst. Stand. Technol. Tech. Note 2144, 17 pages (March 2021)

CODEN: NTNOEF

This publication is available free of charge from: https://doi.org/10.6028/NIST.TN.2144 


\begin{abstract}
User Equipment (UE)-to-Network Relay functionality was introduced in Release 13 of the Third Generation Partnership Project (3GPP) Long Term Evolution (LTE) to extend and improve user connectivity. In this architecture, a UE connected to the network (relay UE) provides network access to another UE (remote UE) by establishing a direct one-toone connection with it using the sidelink (SL) channels over the PC5 interface. The PC5 signalling is used for the establishment and maintenance of this direct connection, and different procedures and timers are linked to these processes. In this paper, we focus on describing and evaluating the PC5 signalling protocol performance through system-level simulations to provide some insights on minimum timer values that may increase the probability of successfully establishing and maintaining the connection between a remote UE and a relay UE.
\end{abstract}

\title{
Key words
}

3GPP; D2D; Device-to-device communication; LTE; ns-3; ProSe; Protocols; Proximitybased services; Relay; Remote. 


\section{Table of Contents}

1 Introduction $\quad 1$

2 Related Work 2

3 LTE UE-to-Network Relay 2

3.1 Sidelink Configuration $\quad 2$

3.2 Relay-Remote Connection 3

3.3 PC5 Signalling Protocol 3

3.3.1 Setup 3

3.3.2 Security 4

3.3.3 Keepalive 5

3.3.4 Release 6

4 Performance Evaluation $\quad 7$

$\begin{array}{lll}4.1 & \text { Scenario } & 7\end{array}$

$\begin{array}{lll}4.2 & \text { Results } & 7\end{array}$

5 Discussion $\quad 10$

6 Conclusion $\quad 11$

$\begin{array}{ll}\text { References } & 11\end{array}$

\section{List of Tables}

Table 1 Scenario Inputs $\quad 7$

\section{List of Figures}

Fig. 1 UE-to-Network relay topology serving 2 remote UEs.

Fig. 2 PC5 signalling protocol procedures for ProSe one-to-one direct communication.

Fig. $3 \mathrm{CDF}$ of the roundtrip time for a single exchange of messages over ProSe direct communication.

Fig. $4 \mathrm{CDF}$ of the total time taken by the direct link setup procedure over ProSe direct communication.

Fig. 5 CDF of the success probability for different timer T4100 values 


\section{Introduction}

Device-to-device (D2D) communication between User Equipment (UEs) was defined in Release 12 of Long Term Evolution (LTE) by the Third Generation Partnership Project (3GPP), under the umbrella of Proximity Services (ProSe). This allows D2D-enabled UEs to communicate directly using the sidelink (SL) over the PC5 interface with minimal to no use of the access network and the evolved NodeB (eNB).

In Release 13 UE-to-Network Relays were introduced in order to enhance and extend the network coverage. In that scope, a UE (remote UE) access the network via an indirect path provided by another in-network UE (relay UE) to which it connects directly using the sidelink. To initiate the relay functionality, the remote UE starts with the discovery and selection of the relay UE according to different criteria. Once the relay is effectively discovered and selected, the one-to-one direct communication connection between a remote UE and a relay UE must be established and maintained to properly ensure the role of relaying traffic between the network and the remote UE.

The PC5 signalling protocol, which is used for the establishment and maintenance of the one-to-one direct communication connection between a relay UE and a remote UE, involves different procedures and timers that are described in the 3GPP standard [1]. One important aspect related to the configuration of the PC5 signaling protocol is setting the values of the retransmission timers, which account for the time a UE waits before retransmitting a message after sending a request without receiving a response. However, $3 \mathrm{GPP}$ standards do not present any recommendation on these timer values and setting them is left to manufacturer implementation.

In this paper, we use system level simulations to evaluate the PC5 signalling message round trip time (RTT) between a remote UE and a relay UE over the sidelink. We then extend the analysis to the direct communication connection establishment procedure, which encompasses multiple PC5 signaling message exchanges, and evaluate the total time taken by the procedure based on different ProSe direct communication configurations. We use this information to obtain a range of potential retransmission timer values for this procedure and finally, we estimate the empiric probability of successfully establishing the connection depending on the different timer values. These results can be used to select minimum values for the corresponding timer so that the reliability of the one-to-one connection increases.

The rest of the paper is organized as follows. In Section 2 we discuss work related to LTE ProSe-based UE-to-Network Relays. In Section 3 we present an overview of the UE-to-Network Relay PC5 Signalling Protocol and its associated timers. Performance evaluation and simulation results are described in Section 4. In Section 5 we summarize the importance of defining adequate timer values for the relay network performance. Finally we conclude our work in Section 6. 


\section{Related Work}

Existing work on UE-to-Network Relays focuses on the relay discovery and selection [2][3][4], end-to-end communication performance [5], and energy efficiency [6][7].

With the increase research on $5 \mathrm{G}$ features, authors in [8] examined relay discovery and selection for 5G D2D communications and proposed a distributed energy-efficient D2D relaying mechanism.

The authors in [9] discussed the challenges related to end-to-end Quality of Service (QoS) support in UE-relayed communications, and how important it is to set the right relay parameters to ensure service continuity. This would allow for a reliable and swift connection establishment.

In [10], the authors evaluated the performance of an off-network mode Mission-CriticalPush-to-Talk (MCPTT) application over ProSe by focusing on the access time, a measure of the delay incurred before a user can talk. An analytical model was developed to calculate the one-way and roundtrip message transmission times over sidelink direct communication. The theoretical model considers the best and worst scenarios and provides only the minimum, maximum, and average values of the RTT over the sidelink.

Given that the PC5 signaling retransmission timers are intrinsically related to the sidelink direct communication RTT, in this paper we use the analytical model introduced in [10] as a baseline. We consider system-level simulations to evaluate the performance of the UEto-Network Relay PC5 signalling protocol by bringing attention to the relay timers and their importance in establishing and maintaining a relay-remote connection. The analytical model serves to validate our approach.

To the best of our knowledge there has been no other discussion about the LTE PC5 signaling retransmission timers used for UE-to-Network Relay and their impact on the overall network performance.

\section{LTE UE-to-Network Relay}

In the LTE UE-to-Network Relay architecture, remote UEs and relay UEs use ProSe direct communication to exchange signaling messages and data packets, and thus the SL pool configuration impacts the UE-to-Network Relay functionality.

\subsection{Sidelink Configuration}

The ProSe direct communication configuration defines multiple parameters including the sidelink (SL) period (which is of configurable length, between $40 \mathrm{~ms}$ and $320 \mathrm{~ms}$ ) and the available resource pool in terms of frequency (resource blocks) and time (subframes) [11]. These resources repeat periodically each SL period, and transmissions occur following a Time Resource Pattern (TRP). A detailed sidelink resource pool analysis can be found in [12]. 


\subsection{Relay-Remote Connection}

The UE-to-Network relay (as the name suggests) is connected to the conventional LTE network and to other UEs using the SL. It relays traffic between remote UEs and the eNB by using SL on the remote side and the Downlink (DL)/Uplink (UL) on the eNB side. A relay UE may extend the network coverage for multiple remote UEs, as illustrated in Figure 1.

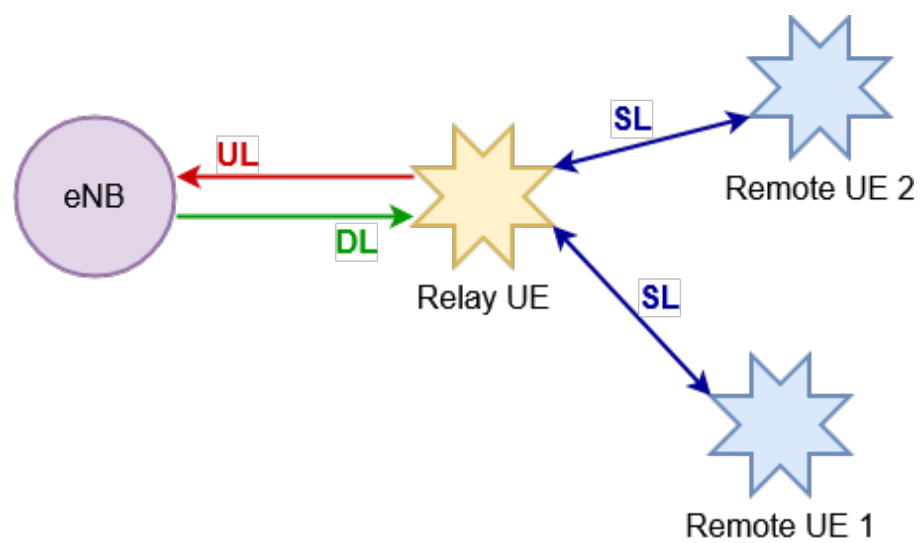

Fig. 1. UE-to-Network relay topology serving 2 remote UEs.

In order to establish this connection, the remote UE starts by looking for nearby relay UEs, then picks one that fits different measures. Once a relay UE is discovered and selected, a one-to-one direct communication link needs to be established, through the PC5 signaling protocol over the sidelink.

\subsection{PC5 Signalling Protocol}

The PC5 signalling protocol procedures between two ProSe-enabled UEs for one-to-one ProSe direct communication are described in [1] and illustrated in Figure 2.

The UE sending the request message is called the "initiating UE" or "requesting UE" and the other UE is called the "target UE". Different PC5 signalling protocol procedures are defined, including the direct link setup, direct link authentication/security, direct link keepalive, and direct link release.

Most of the procedures (e.g., setup, keepalive, and release procedures) are each controlled by one retransmission timer, which defines the wait time for the response message before retransmitting the request. For security, no retransmission is defined and the expiration of the timer initiates the release procedure.

\subsubsection{Setup}

The direct link setup procedure is used to establish a secured direct link between two ProSeenabled UEs. 


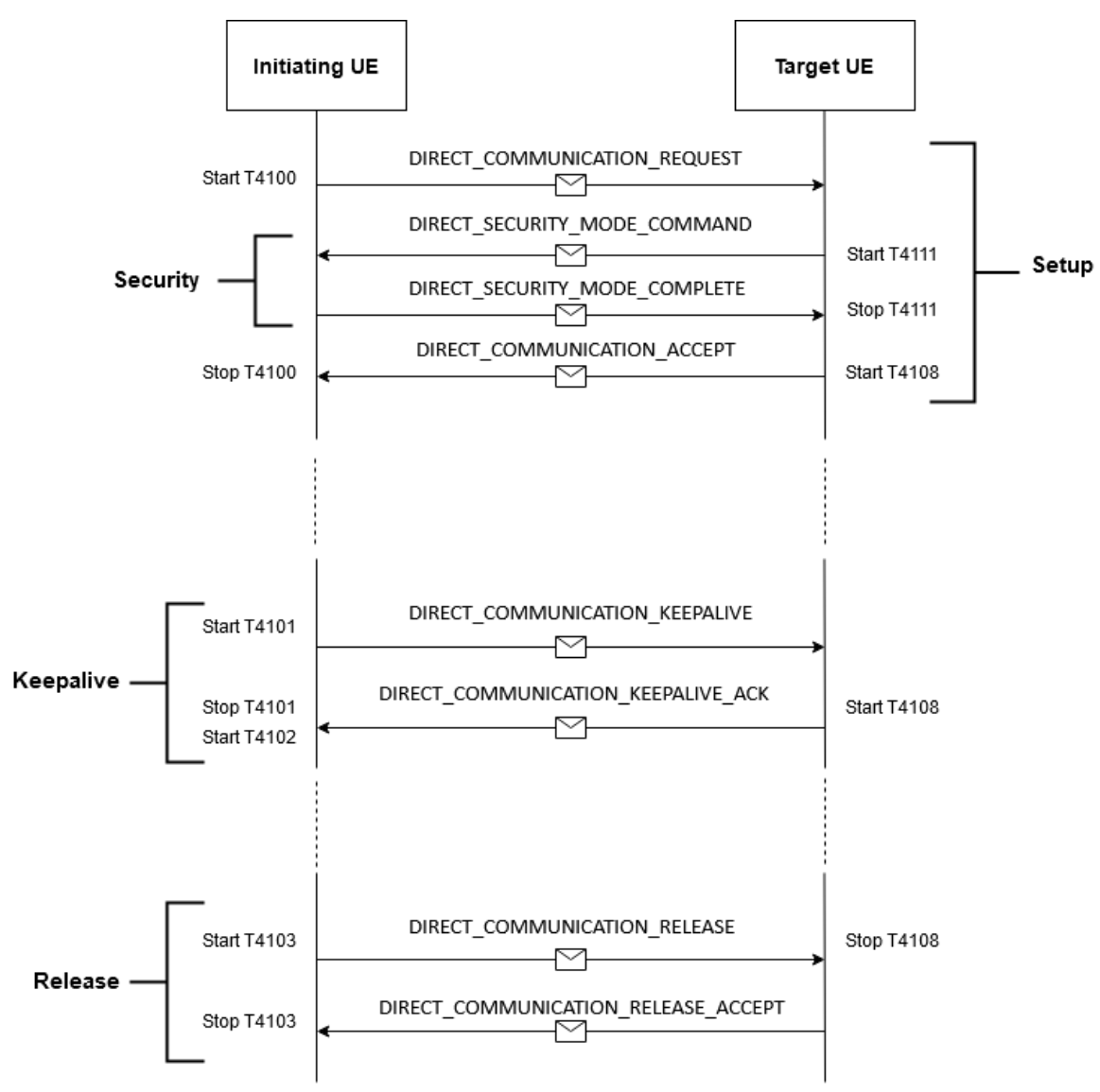

Fig. 2. PC5 signalling protocol procedures for ProSe one-to-one direct communication.

The initiating UE triggers the direct link setup procedure by generating a DIRECT_COMMUNICATION_REQUEST message and starts the timer T4100. After the completion of link security procedure and a successful establishment of the security association, the target UE sends a DIRECT_COMMUNICATION_ACCEPT message to the initiating UE.

Upon the reception of the DIRECT_COMMUNICATION_ACCEPT message, the initiating UE stops the timer T4100. From this time onward, the initiating UE uses the established link for all one-to-one communication (including additional PC5 signalling messages) with the target UE.

\subsubsection{Security}

The security procedure for a direct link between two ProSe-Enabled UEs is performed during the direct link setup procedure or direct link rekeying procedure. It is initiated after the reception of DIRECT_COMMUNICATION_REQUEST or a

DIRECT_REKEYING_REQUEST message.

The UE includes the received UE security capabilities that were present in the DI- 
RECT_COMMUNICATION_REQUEST or a DIRECT_REKEYING_REQUEST message that triggered the DIRECT_SECURITY_MODE_COMMAND message.

The commanding UE sends the DIRECT_SECURITY_MODE_COMMAND message unciphered, but integrity-protected with the new security context. After sending the security request message, the commanding UE starts the timer T4111.

The peer UE checks whether the security mode command can be accepted or not upon reception. This is done by performing the integrity check of the message and by checking that the received UE security capabilities have not been altered compared to the latest values that the peer UE sent to the commanding UE in the

DIRECT_COMMUNICATION_REQUEST or DIRECT_REKEYING_REQUEST message.

If the DIRECT_SECURITY_MODE_COMMAND message can be accepted, the peer UE sends a DIRECT_SECURITY_MODE_COMPLETE (a.k.a security response) message ciphered and integrity-protected with the new security context.

The timer T4111 is stopped after the reception of the DIRECT_SECURITY_MODE_COMPLETE message.

\subsubsection{Keepalive}

The direct link keepalive procedure is used to maintain the direct link between two ProSeenabled UEs and checks if the link is still viable.

An inactivity timer T4108 is considered. Through the keepalive procedure, the requesting UE manages a keepalive timer T4102 and a keepalive counter. T4102 is used to trigger the periodic initiation of the procedure. The timer is started or restarted whenever the UE receives any PC5 communication (i.e., control and data) from the peer UE over this link. The keepalive counter is set to an initial value of zero after link establishment, incremented after the completion of a keepalive procedure.

If the target UE is a ProSe-UE-to-Network Relay UE, the target UE creates an inactivity timer T4108 with the the maximum inactivity period value included in the DIRECT_COMMUNICATION_REQUEST message, and starts the timer T4108 when it has no more messages to send over the link to be established. Once the timer T4108 is started, if any communication activity occurs before the timer T4108 expires, the UE stops the timer T4108 and resets it with the initial value, unless a new value is provided in a DIRECT_COMMUNICATION_KEEPALIVE message. If the timer expires, the ProSe UEto-Network Relay UE initiates the direct link release procedure. In addition, upon receiving a DIRECT_COMMUNICATION_RELEASE message, the peer UE stops all timers related to this link including T4108.

This procedure can be triggered after a request from the upper layers is received or if the keepalive timer T4102 for this link expired.

When the procedure is initiated, the timer T4102 is stopped (if it is still running) and a keepalive request message, named DIRECT_COMMUNICATION_KEEPALIVE, is generated. The message contains the value of the keepalive counter for this link. The keepalive request message will be passed to the lower layers for transmission, at which point a re- 
transmission timer T4101 is started.

Upon the reception of a keepalive request message, the peer UE responds with a keepalive response message, called DIRECT_COMMUNICATION_KEEPALIVE_ACK, including the value of the keepalive counter received in the DIRECT_COMMUNICATION_KEEPALIVE message.

Once the keepalive response message is received, the requesting UE stops retransmission timer T4101, starts keepalive timer T4102, and increments the keepalive counter for this link.

If the retransmission timer T4101 expires, the requesting UE initiates the retransmission of the DIRECT_COMMUNICATION_KEEPALIVE message with the last used keepalive counter value and restarts timer T4101. The action is repeated until a response is received from the peer UE or until the maximum number of allowed retransmissions is reached. In case of failure, the requesting UE aborts the keepalive procedure and proceeds with the release of the link instead.

\subsubsection{Release}

The direct link release procedure is used to release a secure direct link between two ProSeenabled UEs. The link can be released from either end points, due to multiple reasons, including the direct communication to peer UE is no longer needed, no longer allowed, or no longer available.

Once the release request message DIRECT_COMMUNICATION_RELEASE is generated, the releasing UE passes it to the lower layers for transmission. The direct link can be released locally if the direct connection is no longer available. Otherwise, the releasing UE starts the timer T4103.

Upon receiving a release request message, the peer UE stops the timers T4101, T4102, T4103, or T4108 for this link, if any of those timers is running, and abort any other ongoing PC5 Signalling Protocol procedures on this link, and finally responds with a release response message, named DIRECT_COMMUNICATION_RELEASE_ACCEPT.

After reception of a release response message, the releasing UE stops the timer T4103 and no longer sends or receives any messages via this link.

If the retransmission timer T4103 expires, the releasing UE initiates the retransmission of the DIRECT_COMMUNICATION_RELEASE message and restarts T4103. If no response is received after reaching the maximum number of allowed retransmissions, the releasing UE releases the direct link locally, removes the link context, and no longer sends or receives any messages via this link. 


\section{Performance Evaluation}

We performed system-level simulations using the ns-3 ProSe model described in $[5,13]$ (publicly available in [14]), which supports the UE-to-Network Relay capability.

\subsection{Scenario}

We consider a scenario of one Relay UE and one Remote UE and we use the same assumptions as the analytical model in [10], defined in Table 1.

We vary the SL period and run 10000 independent trials per case.

The goal in this study is to evaluate the RTT for one simple request/response (single message exchange), in function of the time and the sidelink period, and both the total time and the success probability for the communication establishment (a more complex exchange of two sets of messages).

Table 1. Scenario Inputs

\begin{tabular}{|l|c|}
\hline Parameter Name & Value \\
\hline Sidelink Period (ms) & 40, 60, 80, 120, 160, 240, 280,320 \\
Control channel duration (ms) & 8 \\
Number of transmission attempts & 4 \\
Time Resource Pattern parameters & 8,1 \\
\hline
\end{tabular}

\subsection{Results}

We plot the cumulative distribution function (CDF) of the RTT for one set of message exchange (request/response) over ProSe for multiple sidelink period values, as shown in Figure 3. In Figure 3a the RTT is measured in function of time (ms) and in Figure 3b, the RTT is calculated in function of the sidelink period (related to the considered period).

The system level simulation results match the theoretical model in [10], which validates the accuracy of both models. However, the analytical model provides only the minimum, maximum, and average values of the RTT over ProSe direct communication, while the simulation results provide a full range of values through the CDFs. They give us a better understanding of the RTT distribution and help us identify any incoherent points.

The results in Figure 3a show that the SL period has a significant impact on the RTT of one request/response over ProSe direct communication, with larger period durations leading to longer RTT values. By normalizing the results using the SL period duration, we observe in Figure $3 b$ that the RTT is directly correlated to the SL duration. We note that the normalization method used produces a slight offset because it does not take into account delays introduced by the control channel which is located at the beginning of the SL period. This offset is higher when the period duration is smaller. The results show that it takes slightly more than one period for the best case scenario, less than 3.2 SL periods for 


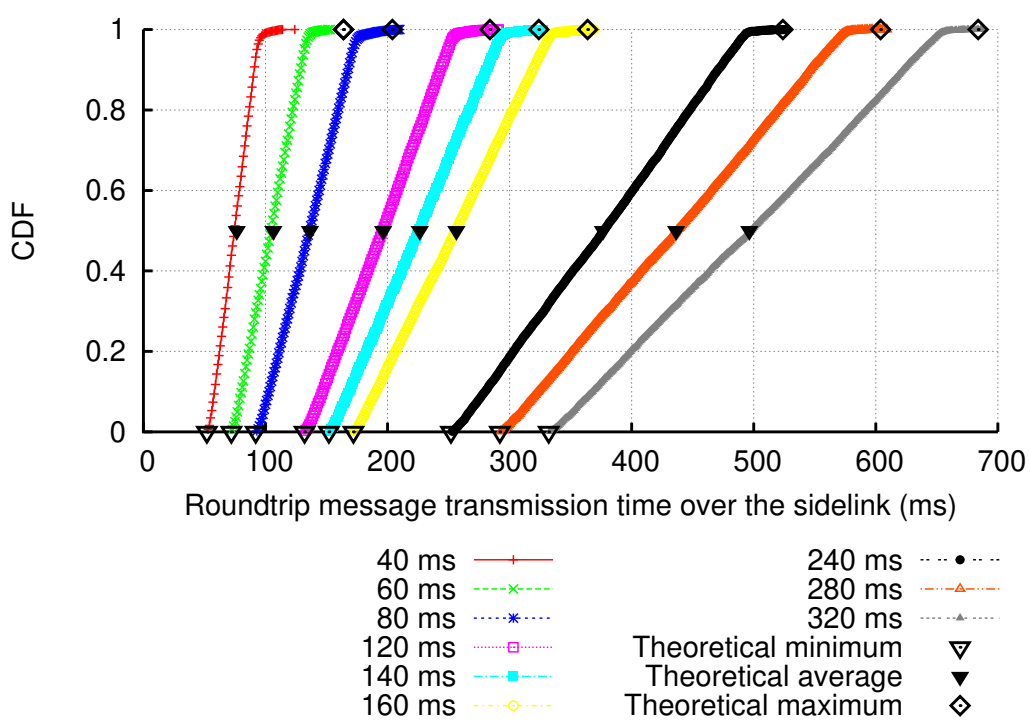

(a) in function of the time (ms)

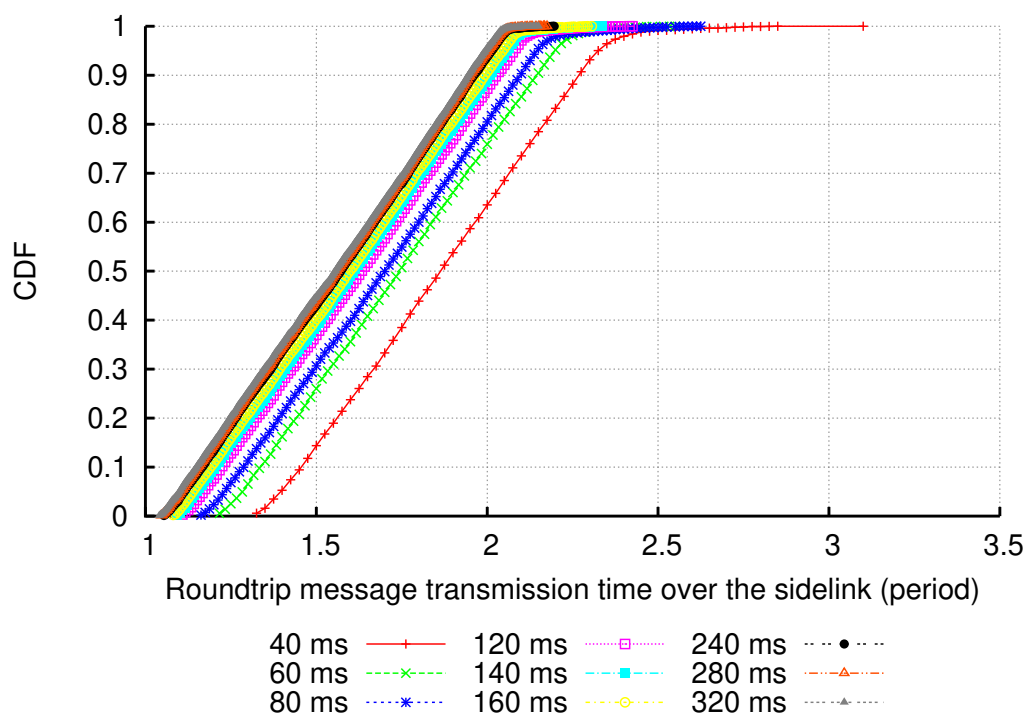

(b) in function of the sidelink period

Fig. 3. CDF of the roundtrip time for a single exchange of messages over ProSe direct communication.

the worst case scenario, and between (1.5 and 1.9) SL periods on average, independently of the sidelink period.

These results would apply for any message exchange over ProSe direct communication. In the case of the PC5 signaling protocol procedures this includes the security (related to the timer T4111), keepalive (related to the timer T4101), and release (related to the timer T4103) procedures. 
The direct link setup procedure does not consider sequential request/response, but one request/response encapsulating another request/response, which are different in terms of latency and processing. That is why we cannot simply double the time of a simple request/response. In Figure 4, we plot the CDFs of the total time taken by the message exchange involved in the direct link setup procedure, for multiple SL period values, in function of time (Figure 4a) and of periods (Figure 4b).

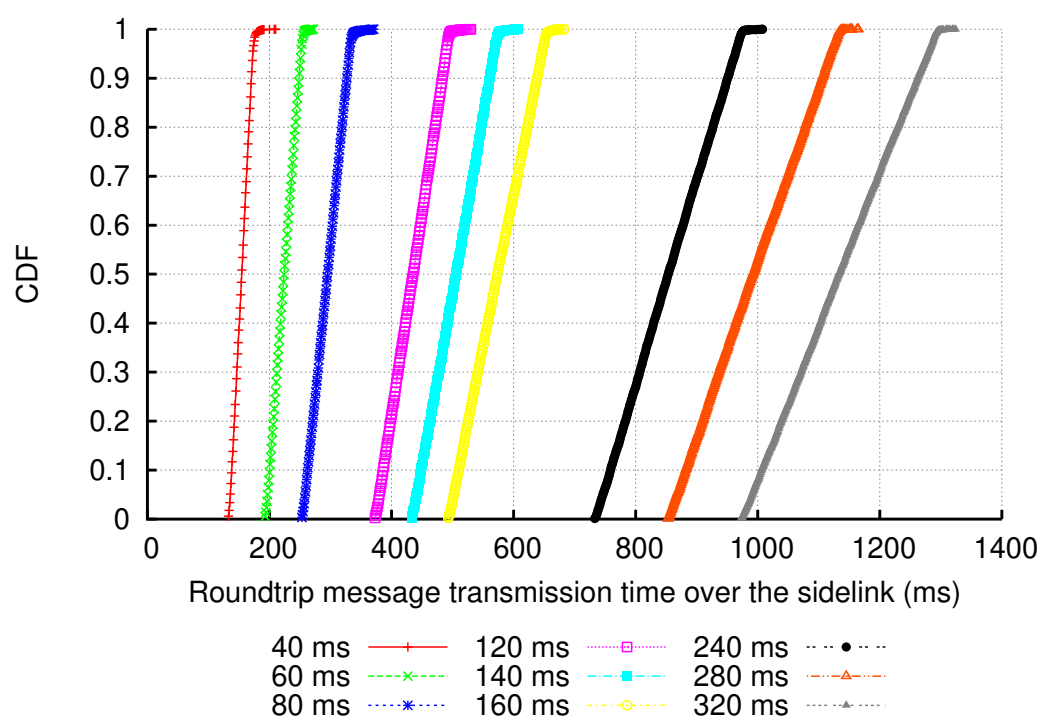

(a) in function of the time (ms)

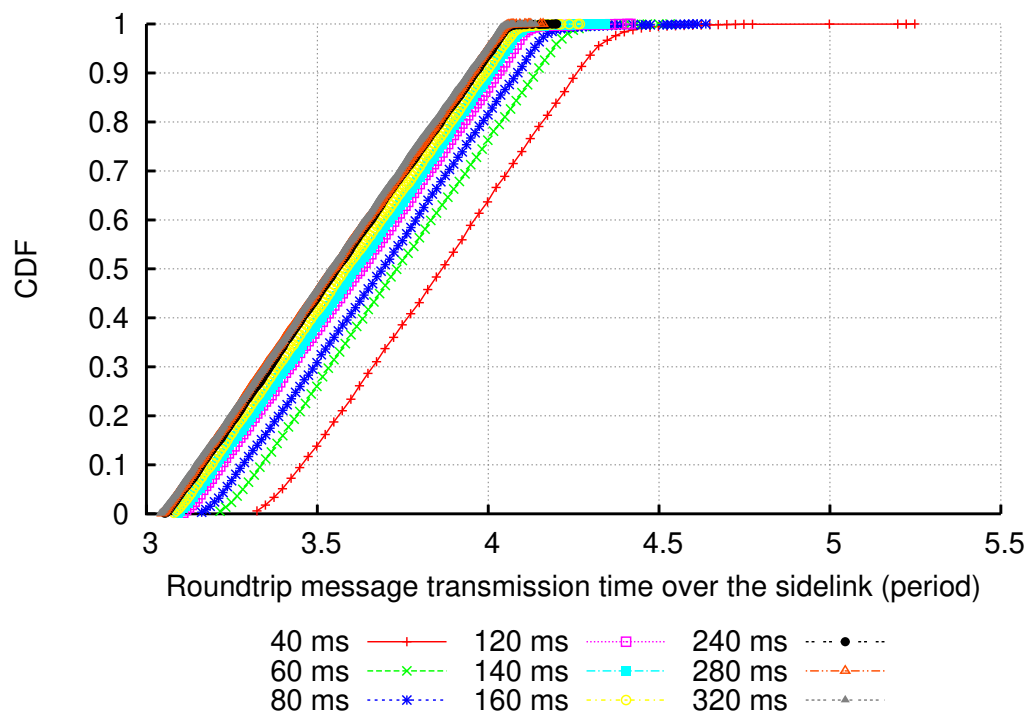

(b) in function of the sidelink period

Fig. 4. CDF of the total time taken by the direct link setup procedure over ProSe direct communication. 
It takes slightly over $3 \mathrm{SL}$ periods (best case scenario) and at most 5.3 SL periods (worst case scenario) to establish a relay-remote connection (which includes 2 sets of request/response messages: one for the security command and another for the communication procedure).

In Figure 5, we vary the timer T4100 value and we plot the success probability of establishing a remote/relay connection for multiple sidelink period values. The RTT and the success probability results are consistent. We have slightly over $99 \%$ success rate if the timer is set to 4.5 SL periods, independently of the sidelink period values.

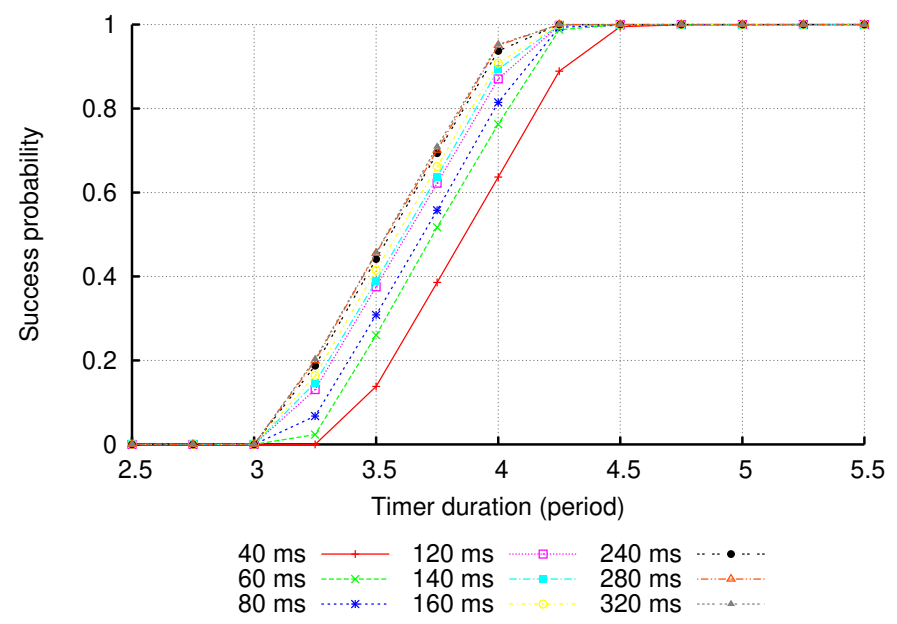

Fig. 5. CDF of the success probability for different timer T4100 values

\section{Discussion}

The simulation results provide the CDF of the roundtrip time over ProSe for all possible SL periods.

For the security procedure, it is important to define the timer appropriately since no retransmission is permitted. Otherwise the direct communication setup would be aborted.

In general, setting the timer to a smaller value than the RTT would waste energy and resources due to unnecessary retransmissions. We may also exhaust all retransmissions before getting a response, and ineffectively clutter the channel. However, using a larger value than the RTT time may slow down the process and create unwanted latency.

Based on the results shown in the previous section, we observe that the timer value for one request/response over ProSe should be at least 2.5 SL periods, while for the setup timer, we would suggest at least $4.5 \mathrm{SL}$ periods. Those values should be considered as lower bound values because we only assumed one pair of remote/relay UEs. In addition, the UEs are close enough and the signal strength is high enough that no packet loss is experienced. When there are more nodes, the RTT is likely to increase due to interference and/or scheduling policies, and timer values may need to be increased as well. 
Recently, while defining unicast communication in Vehicle-to-Everything (V2X) services in 5G System (5GS), 3GPP provided some values to similar timers in Release 16 [15]. However, those values are static. And as we saw in Section 4 for LTE ProSe, the values depend of the SL period length and eventually on the number of remotes and relays in the system.

While our model captures the roundtrip time and success probability for a successful remote/relay communication establishment, it did not take into account the potential effect of having multiple remotes trying to connect to one (or multiple) relay(s). This would involve other simulation details, e.g. physical channel model and resource allocation algorithm, which would affect the above-mentioned results. We focused on a single communication link as it will lay the groundwork for further studies.

\section{Conclusion}

In this paper, we considered LTE UE-to-Network Relays and evaluated the PC5 signalling protocol performance. We highlighted the importance of setting retransmission timers to the appropriate values and we provided lower bounds that can enhance the probability of a successful one-to-one connection between a remote and a relay UE.

This work is the baseline for such studies and our simulation model can help simulate more complex scenarios to guide the configuration of the timers. For future research, it would be insightful to extend this paper by considering multiple remote UEs per relay UE.

\section{References}

[1] 3GPP (2020) Proximity-services (ProSe) User Equipment (UE) to ProSe function protocol aspects (Third Generation Partnership Project (3GPP)), TS 24.334. Available at http://www.3gpp.org/ftp/Specs/html-info/24334.htm.

[2] Gamboa S, Moreaux A, Griffith D, Rouil R (2020) UE-to-Network Relay Discovery in ProSe-enabled LTE Networks. 2020 International Conference on Computing, Networking and Communications (ICNC) (IEEE), , pp 871-877. https://doi.org/ 10.1109/ICNC47757.2020.9049657

[3] Wang J, Xu X, Tang X, Zhang S, Tao X (2018) Analytical Modeling of Mode Selection for UE-To-Network Relay Enabled Cellular Networks with Power Control. 2018 IEEE International Conference on Communications Workshops (ICC Workshops) (IEEE), , pp 1-6. https://doi.org/10.1109/ICCW.2018.8403501

[4] Huang J, Fang D, Ye F, Hu RQ, Qian Y (2018) A Relay Selection Scheme to Prolong Connection Time for Public Safety Communications. 2018 IEEE 87th Vehicular Technology Conference (VTC Spring) (IEEE), , pp 1-5. https://doi.org/10.1109/ VTCSpring.2018.8417847

[5] Gamboa S, Thanigaivel R, Rouil R (2019) System Level Evaluation of UE-toNetwork Relays in D2D-Enabled LTE Networks. 2019 IEEE 24th International Work- 
shop on Computer Aided Modeling and Design of Communication Links and Networks (CAMAD) (IEEE), , pp 1-7. https://doi.org/10.1109/CAMAD.2019.8858441

[6] Zhang S, Xu X, Sun M, Tang X, Tao X (2017) Energy efficient uplink transmission for ue-to network relay in heterogeneous networks. 2017 IEEE 28th Annual International Symposium on Personal, Indoor, and Mobile Radio Communications (PIMRC) (IEEE), , pp 1-7. https://doi.org/10.1109/PIMRC.2017.8292436

[7] Chaudhari A, Gandikota J, Sen A, Narayan S (2020) A Realistic Approach to Enhance the Battery Performance of Device-to-Device (D2D) Relay UEs. 2020 IEEE 17th Annual Consumer Communications \& Networking Conference (CCNC) (IEEE), , pp 1-2. https://doi.org/10.1109/CCNC46108.2020.9045552

[8] Anamuro CV, Varsier N, Schwoerer J, Lagrange X (2021) Distance-aware relay selection in an energy-efficient discovery protocol for 5G D2D communication. IEEE Transactions on Wireless Communications https://doi.org/10.1109/TWC.2021. 3058636

[9] Kozioł D, Moya FS, Yu L, Van Phan V, Xu S (2017) QoS and service continuity in 3GPP D2D for IoT and wearables. 2017 IEEE Conference on Standards for Communications and Networking (CSCN), , pp 233-239. https://doi.org/10.1109/CSCN. 2017.8088627

[10] Sun Y, Garey W, Rouil R, Varin P (2019) Access Time Analysis of MCPTT OffNetwork Mode over LTE. Wireless Communications and Mobile Computing 2019. https://doi.org/10.1155/2019/2729370

[11] 3GPP (2020) Evolved Universal Terrestrial Radio Access (E-UTRA); Physical layer procedures (Third Generation Partnership Project (3GPP)), TS 36.213. Available at http://www.3gpp.org/ftp/Specs/html-info/36213.htm.

[12] Griffith DW, Cintrón FJ, Rouil RA (2017) Physical sidelink control channel (PSCCH) in mode 2: Performance analysis. 2017 IEEE International Conference on Communications (ICC) (IEEE), , pp 1-7. https://doi.org/10.1109/ICC.2017.7997074

[13] Rouil R, Cintrón FJ, Ben Mosbah A, Gamboa S (2017) Implementation and Validation of an LTE D2D Model for ns-3. Proceedings of the Workshop on $n s-3$ WNS3 '17, , pp 55-62. https://doi.org/10.1145/3067665.3067668

[14] NIST (2020) Public Safety Communication modeling tools based on ns-3. Available at https://github.com/usnistgov/psc-ns3.

[15] 3GPP (2020) Vehicle-to-Everything (V2X) services in 5G System (5GS); Stage 3 (Third Generation Partnership Project (3GPP)), TS 24.587. Available at http://www. 3gpp.org/ftp/Specs/html-info/24587.htm. 